\title{
From Hydrodynamics to Jet Quenching, Coalescence, and Hadron Cascade: A Coupled Approach to Solving the $R_{A A} \otimes v_{2}$ Puzzle
}

\author{
Wenbin Zhao $\odot,{ }^{1}$ Weiyao Ke, ${ }^{2,3}$ Wei Chen $\odot,{ }^{4}$ Tan Luo, ${ }^{5}$ and Xin-Nian Wang $\oplus^{1,3, *, \dagger}$ \\ ${ }^{1}$ Key Laboratory of Quark and Lepton Physics (MOE) and Institute of Particle Physics, Central China Normal University, \\ Wuhan 430079, China \\ ${ }^{2}$ Physics Department, University of California, Berkeley, California 94720, USA \\ ${ }^{3}$ Nuclear Science Division MS 70R0319, Lawrence Berkeley National Laboratory, Berkeley, California 94720, USA \\ ${ }^{4}$ School of Nuclear Science and Technology, University of Chinese Academy of Sciences, Beijing 100049, China \\ ${ }^{5}$ Instituto Galego de Física de Altas Enerxías IGFAE, Universidade de Santiago de Compostela, E-15782 Galicia-Spain
}

(Received 28 April 2021; revised 2 August 2021; accepted 24 December 2021; published 14 January 2022)

\begin{abstract}
Hydrodynamics and jet quenching are responsible for the elliptic flow $v_{2}$ and suppression of large transverse momentum $\left(p_{T}\right)$ hadrons, respectively, two of the most important phenomena leading to the discovery of a strongly coupled quark-gluon plasma in high-energy heavy-ion collisions. A consistent description of the hadron suppression factor $R_{A A}$ and $v_{2}$, especially at intermediate $p_{T}$, however, remains a challenge. We solve this long-standing $R_{A A} \otimes v_{2}$ puzzle by including quark coalescence for hadronization and final state hadron cascade in the coupled linear Boltzmann transport-hydro model that combines concurrent jet transport and hydrodynamic evolution of the bulk medium. We illustrate that quark coalescence and hadron cascade, two keys to solving the puzzle, also lead to a splitting of $v_{2}$ for pions, kaons, and protons in the intermediate $p_{T}$ region. We demonstrate for the first time that experimental data on $R_{A A}, v_{2}$, and their hadron flavor dependence from low to intermediate and high $p_{T}$ in high-energy heavy-ion collisions can be understood within this coupled framework.
\end{abstract}

DOI: 10.1103/PhysRevLett.128.022302

Introduction.-Experimental evidences at the Relativistic Heavy-Ion Collider (RHIC) and the Large Hadron Collider (LHC) have confirmed the existence of a strongly coupled quark-gluon plasma (QGP) in high-energy heavy-ion $(A+A)$ collisions [1-5]. These include strong anisotropic flow at low transverse momentum $\left(p_{T}\right)$ [6-10] and suppression of hadrons at high $p_{T}$ [11-15]. The spectra and flow patterns of bulk hadrons at low $p_{T} \lesssim 2 \mathrm{GeV} / c$ are well described by the hydrodynamic expansion of the QGP as a strongly coupled fluid [16-25]. At high $p_{T} \gtrsim$ $10 \mathrm{GeV} / c$, the hadron suppression factor $\left(R_{A A}\right)$ and the azimuthal anisotropy $\left(v_{2}\right)$ [26-31] can be quantitatively understood in terms of jet quenching caused by parton energy loss as hard partons propagate through the QGP medium [32-48]. In the intermediate $p_{T} \sim 2-10 \mathrm{GeV} / c$ region where soft and hard physics interface it remains, however, a challenge to describe the hadron spectra and the azimuthal anisotropy consistently. There is a long-standing puzzle that parton energy loss models that are adjusted to describe hadron $R_{A A}$ underpredict the azimuthal anisotropy

Published by the American Physical Society under the terms of the Creative Commons Attribution 4.0 International license. Further distribution of this work must maintain attribution to the author(s) and the published article's title, journal citation, and DOI. Funded by SCOAP ${ }^{3}$. $v_{2}$ [49-55] at intermediate $p_{T}$. Parton energy loss alone also cannot describe the constituent quark number (NCQ) dependence of hadron $R_{A A}$ and $v_{2}$.

Many attempts have been made to solve this puzzle, from evoking the exotic monopoles in interactions between the hard partons and the medium near the pseudocritical temperature $T_{c}$ [56-58] to taking into account of the event-by-event fluctuation of the bulk medium [50] and combining hydrodynamics with jet transport as in EPOS [59] and HYDJET++ models [60]. While such an exotic interaction with a drastically large jet-medium coupling at $T_{c}$ is not needed to describe $R_{A A}$ and $v_{2}$ of full jets [61-64] in which a hadron's flavor information is not considered, the event-byevent fluctuation of the bulk medium is found not to significantly increase high $p_{T} v_{2}$ [54,64,65]. Furthermore, none of these attempts can address the NCQ dependence of $R_{A A}$ and $v_{2}$ in the intermediate $p_{T}$ region.

It is well known that quark coalescence is the key to explaining the observed NCQ scaling of hadronic anisotropies and the enhanced baryon-to-meson ratios in $A+A$ relative to proton-proton $(p+p)$ collisions at intermediate $p_{T}$ [66-78]. Furthermore, it has been shown that hadrons, especially baryons, at intermediate $p_{T}$ are also sensitive to rescatterings in the hadronic phase [79-81]. Quark coalescence in a hydrodynamic medium combined with parton transport, fragmentation and hadron cascade should, therefore, have the potential to solve the $R_{A A} \otimes v_{2}$ puzzle and 
describe hadron production from low to intermediate and high $p_{T}$ in high-energy heavy-ion collisions.

In this Letter, we implement the quark coalescence, especially between thermal and jet shower partons, and a hadronic afterburner in the state-of-the-art coupled linear Boltzmann transport(COLBT)-hydro model [82] that has concurrent evolution of both the bulk medium and jet showers, including jet-induced medium responses. We carry out a first study that couples event-by-event hydrodynamics, jet quenching, quark coalescence, and hadron cascade. We demonstrate that this fully coupled approach can simultaneously describe $R_{A A}$, differential $v_{2}$, and their NCQ dependence in the full range of $p_{T}$ in high-energy heavy-ion collisions, therefore solving the long-standing $R_{A A} \otimes v_{2}$ puzzle that connects the two most important aspects of the discovery of a strongly coupled QGP. This also sheds light on the hadronization mechanism of QGP in high-energy heavy-ion collisions.

COLBT-hydro model.-The COLBT-hydro model [82-84] was developed to simulate the concurrent evolution of jet showers and the bulk medium by coupling the $(3+1) \mathrm{D}$ CCNU (Central China Normal University) LBNL (Lawrence Berkeley National Laboratory) viscous hydrodynamic model (CLVISC) [85,86] with the linear Boltzmann transport (LBT) model [87-91]. The LBT model treats the propagation of jet shower and thermal recoil partons on an equal footing and includes both perturbative QCD (PQCD) elastic scattering and medium-induced gluon radiation within the high-twist approach [92-95]. The coupling between LBT and CLVISC is through an energy-momentum source term deposited by soft partons in the hydrodynamic equation,

$$
\begin{gathered}
\partial_{\mu} T_{\text {fluid }}^{\mu \nu}=J^{\nu} \\
J^{\nu}=\sum_{i} \frac{\theta\left(p_{\text {cut }}^{0}-p_{i} \cdot u\right) p^{\nu}}{\tau(2 \pi)^{3 / 2} \sigma_{r}^{2} \sigma_{\eta_{s}} \Delta \tau} e^{-\frac{\left(\vec{x}_{\perp}-\vec{x}_{\perp i}\right)^{2}}{2 \sigma_{r}^{2}}-\frac{\left(\eta_{s}-\eta_{s i}\right)^{2}}{2 \sigma_{\eta_{s}}^{2}}}
\end{gathered}
$$

where $T_{\text {fluid }}^{\mu \nu}$ is the energy-momentum tensor of the bulk medium, and the summation in the Gaussian smearing in the Milne coordinates is over both soft recoil, radiated, and "negative" partons- "hole particles" created in elastic jetmedium collisions. For studies presented in this Letter, we set $p_{\text {cut }}^{0}=3.0 \mathrm{GeV} / c, \sigma_{r}=0.6 \mathrm{fm}$, and $\sigma_{\eta_{s}}=0.6$. The jetinduced medium response is found essential to describing many jet observables in heavy-ion collisions, such as jet shape [96], jet fragmentation function [82], $\gamma / Z$-hadron correlations [83,84], and baryon-to-meson ratio in and around a jet $[97,98]$. However, it has negligible influence on low $p_{T}$ single inclusive hadron spectra due to the dominance of soft particles from the bulk medium.

In the CLVISC hydrodynamic evolution, a lattice QCD inspired equation of state [99] is used. The reduced thickness event-by-event nuclear topology (TRENTO) model [100] with optimized parameters [23] and a longitudinal envelope function $[85,86,101]$ is used to generate the initial entropy density profile with event-by-event transverse fluctuation. The specific shear viscosity $\eta / s=0.10$, the freeze-out temperature $T_{\mathrm{sw}}=150 \mathrm{MeV}$, the initial time $\tau_{0}=0.6 \mathrm{fm} / c$, and parameters in the initial entropy profile have been adjusted to reproduce the charged hadron multiplicity, $p_{T}$ spectra, and integrated flow harmonics $v_{n}$ at midrapidity in $A+A$ collisions. While a finite starting time $\tau_{0}$ of the jet-medium interaction is a default assumption in LBT and COLBT-hydro, other studies $[45,55]$ find it necessary to achieve $v_{2}$ at high $p_{T}$ that is compatible with experimental data. We also assume that hard shower partons free-stream during the formation time $\tau_{f}=2 z(1-z) E / k_{\perp}^{2}$ before they interact with the QGP medium, where $k_{\perp}$ is the transverse momentum, and $z$ is the energy fraction of the shower parton after the initial splitting from its mother parton with energy $E$. Further details about the LBT and CLVISC model can be found in Refs. [87-91] and [85,86], respectively.

We use PYTHIA8 [102] with EPPS16 nuclear parton distributions [103] to generate initial jet showers. A minimum hard scale $\hat{p}_{T 0}=4.0 \mathrm{GeV} / c$ is set for jet production. The average number of such jet production per event is $\left\langle N_{\text {jet }}\right\rangle=\left\langle N_{\text {coll }}\right\rangle P_{\text {trigger }}^{\text {jet }}$, where $\left\langle N_{\text {coll }}\right\rangle$ is the number of binary nucleon-nucleon $(N+N)$ collisions whose transverse distribution is given by the nuclear overlap function. $P_{\text {trigger }}^{\mathrm{jet}}$ is the probability for a minimum-bias $N+N$ collision to have at least one pair of jet production,

$$
P_{\text {trigger }}^{\text {jet }}=\frac{1}{\sigma_{N N}^{\text {inel }}} \int d^{2} b\left[1-e^{-T_{N N}(b) \sigma_{\text {jet }}\left(\hat{p}_{T}>\hat{p}_{T 0}\right)}\right],
$$

where $T_{N N}(b)$ is the nucleon overlapping function and $\sigma_{N N}^{\text {inel }}=70 \mathrm{mb}$ at $\sqrt{s_{N N}}=5.02 \mathrm{TeV}$.

There are two adjustable parameters in this study of single inclusive hadron spectra using the COLBT-hydro model. While the strong coupling $\alpha_{\mathrm{s}}$ for jet production and showering is allowed to run according to PQCD in PYTHIA [90,91], the effective coupling to the medium partons $\alpha_{\mathrm{s}}=$ 0.17 is adjusted to fit $R_{A A}$ at high $p_{T}$ in central $\mathrm{Pb}+\mathrm{Pb}$ collisions at $\sqrt{s_{N N}}=5.02 \mathrm{TeV}$. The second parameter is a lower cutoff $p_{T \min }$ for initial jet partons that propagate according to LBT. Initial partons below this scale are assumed to be thermalized as part of the initial condition for the hydro evolution of the bulk medium. The value $p_{T \min }=$ $7.0(5.0) \mathrm{GeV} / c$ is tuned by fitting the final hadron spectra at intermediate $p_{T}$ in $10 \%-20 \%(40 \%-50 \%)$ central $\mathrm{Pb}+\mathrm{Pb}$ collisions at $\sqrt{s_{N N}}=5.02 \mathrm{TeV}$. Its value depends on the centrality and colliding energy.

Hadronization and hadron cascade.-In this study, we adapt the hydro-coal-frag hybrid model [104] for hadronization that includes hydro freeze-out at low $p_{T}$, quark coalescence at intermediate $p_{T}$, and fragmentation at high 
$p_{T}$. The interplay between hadron freeze-out in hydro and parton dynamics is defined by a separation scale $p_{T s}=$ $1.5 \mathrm{GeV} / c$ for the effective constituent quarks, above which viscous corrections to the equilibrium distribution become large and parton coalescence and fragmentation become the relevant mechanisms for hadronization. This scale corresponds to $p_{\text {Tmeson }}<3 \mathrm{GeV} / c$ and $p_{\text {Tbaryon }}<$ $4.5 \mathrm{GeV} / c$ for hadron production through hydro freeze-out on the switching hypersurface. Accordingly, thermal quarks with $p_{T}>1.5 \mathrm{GeV} / c$ on the switching hypersurface [105] are allowed to participate in coalescence processes for hadronization, which include thermal-thermal, thermal-shower, and shower-shower coalescence. Shower partons include both jet shower and hard medium recoil partons passing through the isothermal hypersurface in COLBT-hydro.

Shower partons that do not coalesce will hadronize through string fragmentation using PYTHIA8 [102] with tuned strangeness suppression [106]. We adopt a colorless hadronization scheme [108] for these shower partons that should have lost their original color configurations and form strings with the distances $\Delta R=\sqrt{(\Delta \eta)^{2}+(\Delta \phi)^{2}}$ of neighboring parton pairs minimized. Finally, the ultrarelativistic quantum molecular dynamics (URQMD) $[109,110]$ model is used to perform hadronic rescatterings and resonance decays in the hadronic stage of the system until kinetic freeze-out.

Nuclear modification of hadron spectra.-Using COLBT-hydro with the inclusion of quark coalescence and hadronic afterburner, we calculate hadron spectra from low to intermediate and high $p_{T}$ in $A+A$ collisions. Shown in Fig. 1 are COLBT-hydro results on the nuclear modification factor $R_{A A}\left(p_{T}\right)$ of charged hadron spectra [111] which describe reasonably well the experimental data [112] for $10 \%-20 \%$ and $40 \%-50 \% \mathrm{~Pb}+\mathrm{Pb}$ collisions at $\sqrt{s_{N N}}=5.02 \mathrm{GeV}$. Only statistical uncertainties in the COLBT-hydro results are shown as solid bands. To illustrate the different hadron production mechanisms

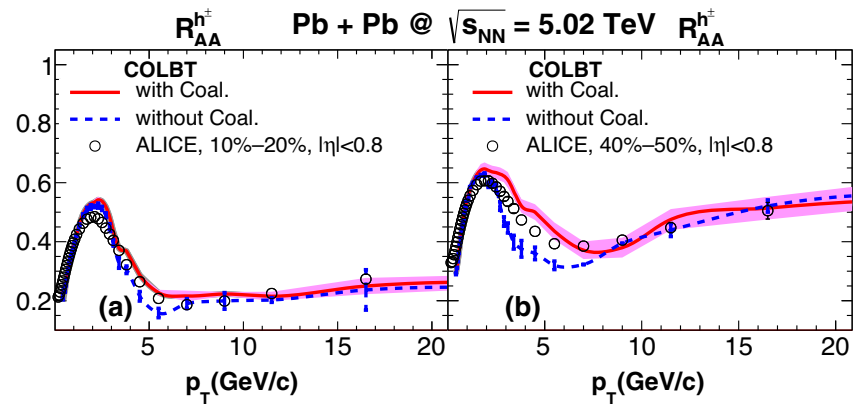

FIG. 1. The nuclear modification factor $R_{A A}$ of charged hadrons in (a) $10 \%-20 \%$ and (b) $40 \%-50 \% \mathrm{~Pb}+\mathrm{Pb}$ collisions at $\sqrt{s_{N N}}=5.02 \mathrm{TeV}$ from COLBT-hydro simulations with (solid) and without quark coalescence(dashed) as compared to ALICE experimental data [112]. underlying $R_{A A}\left(p_{T}\right)$ in different $p_{T}$ regions, we show in Fig. 2(a) $p_{T}$ spectra of charged hadrons in $10 \%-20 \%$ and $40 \%-50 \%$ and Fig. 2(b) identified pion $(\pi)$, kaon $(K)$, and proton $(p)$ spectra from COLBT-hydro in 40\%-50\% $\mathrm{Pb}+\mathrm{Pb}$ collisions as compared to the experimental data $[112,113]$. Also shown are contributions from hydro (dashed), parton coalescence (dot-dashed), and fragmentation (dotted) in COLBT results.

In COLBT-hydro, hadron spectra are dominated by the hydro contribution below $p_{T}<2 \mathrm{GeV} / c$, where $R_{A A}$ increases rapidly with $p_{T}$ due to the strong radial flow from the hydrodynamic expansion until it peaks at $p_{T} \approx 2 \mathrm{GeV} / c$. At intermediate $2<p_{T}<6 \mathrm{GeV} / c, R_{A A}$ decreases from its peak value due to the onset of contributions from parton coalescence and fragmentation in which hadron spectrum are suppressed due to parton energy loss. At large $p_{T}>8 \mathrm{GeV} / c$ where fragmentation prevails, $R_{A A}$ is determined by the energy dependence of the parton energy loss and the initial jet spectra.

We note that the transition in the underlying hadron production mechanism, from hydrodynamics to parton coalescence and fragmentation, occurs at higher $p_{T}$ in more central collisions because of stronger radial flow, pushing the realm of hydrodynamics to higher $p_{T}$. The transition also happens at larger $p_{T}$ for baryons than mesons. This is because of the constituent-quark-based separate scale $p_{T s}=1.5 \mathrm{GeV} / c$ that leads to a larger cutoff $p_{\text {Tbaryon }}<4.5 \mathrm{GeV} / c$ for baryons than $p_{T \text { meson }}<$ $3 \mathrm{GeV} / c$ for mesons from hydro. The hydro spectra are also mass ordered due to radial flow. Hydro contributions to the hadron spectra above the $p_{T}$ cutoff come from hadron cascade within URQMD during the hadronic evolution. The interplay between hydro, parton energy loss, coalescence, and fragmentation implemented in COLBT-hydro

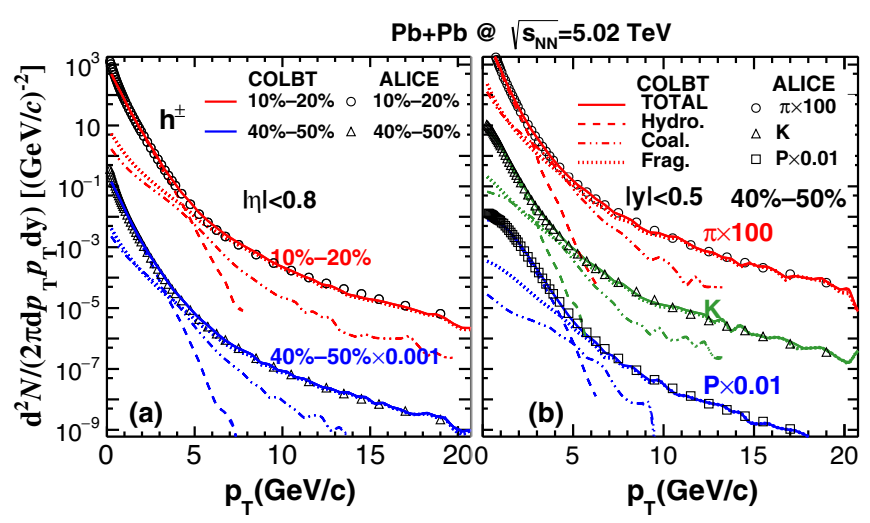

FIG. 2. Spectra of (a) charged hadrons (solid) in 10\%-20\% and $40 \%-50 \%$ and (b) identified $\pi, K$, and $p$ (solid) in $40 \%-50 \%$ $\mathrm{Pb}+\mathrm{Pb}$ collisions at $\sqrt{s_{N N}}=5.02 \mathrm{TeV}$ and contributions from hydro freeze-out (dashed), parton coalescence (dot-dashed), and fragmentation (dotted) in COLBT-hydro simulations as compared to ALICE experimental data [112,113]. 


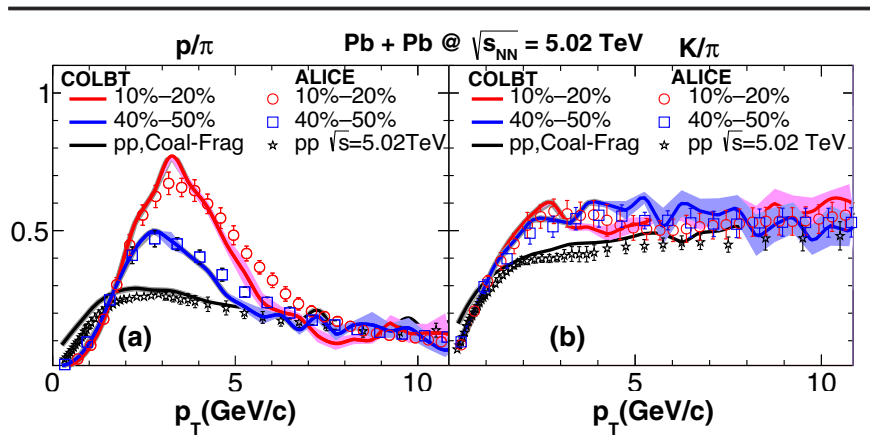

FIG. 3. (a) $p / \pi$ and (b) $K / \pi$ ratio in $10 \%-20 \%$ and $40 \%-50 \%$ $\mathrm{Pb}+\mathrm{Pb}$ and $p+p$ collisions at $\sqrt{s_{N N}}=5.02 \mathrm{TeV}$ from COLBT-hydro as compared to experimental data [113].

can describe the flavor dependence of hadron spectra and their medium modification as seen in Fig. 2(b).

To examine the flavor composition of the hadron spectra and their $p_{T}$ dependence in detail, we compare the COLBT-hydro results on $p / \pi$ and $K / \pi$ ratios as a function of $p_{T}$ to the experimental data in Fig. 3. Both $p / \pi$ and $K / \pi$ ratios exhibit a steep increase from $p_{T}=$ $0-3 \mathrm{GeV} / c$ with a mass ordering induced by the radial flow, an intrinsic feature of hydrodynamic models. For $p_{T}>3 \mathrm{GeV} / c, p / \pi$ decreases with $p_{T}$ while $K / \pi$ remains constant as a result of the interplay between hydrodynamic expansion, quark coalescence, and fragmentation. At large $p_{T}>8 \mathrm{GeV} / c$, these hadron ratios in $\mathrm{Pb}+\mathrm{Pb}$ approach the values in $p+p$ collisions when the hadronization is dominated by vacuumlike parton fragmentation. COLBThydro describes these features in the data well.

Coalescence and $R_{A A} \otimes v_{2}$ puzzle.-To demonstrate the importance of parton coalescence in resolving the $R_{A A} \otimes v_{2}$ puzzle, we examine first the azimuthal anisotropy $v_{2}$ of the hadron spectra for different hadron species. Following the experiments $[27,29,114]$, we use the scalar-product (SP) method to compute $v_{2}$ of charged hadrons within $|\eta|<1.0$, using reference particles in $|\eta|<1.0$ and $0.2<p_{T}<5.0 \mathrm{GeV} / c$.

Shown in Fig. 4 are $v_{2}\left(p_{T}\right)$ for $\pi, K$, and $p$ from COLBT-hydro with (solid) and without (dashed) contributions from coalescence in 10\%-20\% and 40\%-50\% $\mathrm{Pb}+\mathrm{Pb}$ collisions as compared to the experimental data [114]. The interplay among hydro, jet quenching, coalescence, and fragmentation in $v_{2}\left(p_{T}\right)$ is very similar to that in $R_{A A}\left(p_{T}\right)$. To help understand this interplay, we also plot in Fig. $5 v_{2}\left(p_{T}\right)$ for $\pi, K$, and $p$ from hydro freeze-out (dotted), parton fragmentation (dashed), and quark coalescence (solid) which has a strong flavor dependence.

The rapid increase and the mass ordering of $v_{2}\left(p_{T}\right)$ from hydro at low $p_{T}<2 \mathrm{GeV} / c$ in Figs. 4 and 5 are characteristics of viscous hydrodynamics with hadronic afterburner [19-21,24,25,80]. The increase slows down at $p_{T}>2.5 \mathrm{GeV} / c$, and the total $v_{2}\left(p_{T}\right)$ reaches a peak value at around $p_{T \text { meson }} \sim 3 \mathrm{GeV} / c$ and $p_{\text {Tbaryon }} \sim 4.5 \mathrm{GeV} / c$

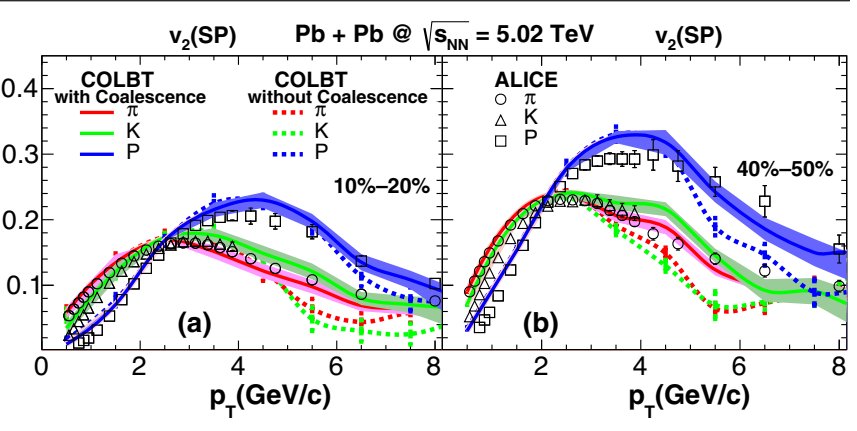

FIG. 4. COLBT-hydro results on $v_{2}\left(p_{T}\right)$ for $\pi, K$, and $p$ in (a) $10 \%-20 \%$ and (b) $40 \%-50 \% \mathrm{~Pb}+\mathrm{Pb}$ collisions at $\sqrt{s_{N N}}=$ $5.02 \mathrm{TeV}$ with (solid) and without (dashed) contributions from quark coalescence compared to ALICE data [114].

before decreasing at large $p_{T}$, where the anisotropy caused by the geometric anisotropy of the QGP fireball and the length dependence of parton energy loss is significantly smaller than that from hydro and parton coalescence. The mass ordering is also reversed starting at $p_{T} \approx$ $2.5 \mathrm{GeV} / c$. The change of $p_{T}$ dependence and mass ordering of $v_{2}\left(p_{T}\right)$ are caused mainly by the interplay among hadrons from hydro, coalescence, and fragmentation. Since the transition from hydro to coalescence and fragmentation occurs at a fixed $p_{T s}$ for the effective constituent quarks, both the value and the peak position of the final $v_{2}$ for $p$ is higher than $\pi$ and $K$ in the intermediate $3<p_{T}<$ $8 \mathrm{GeV} / c$ region, resembling the approximate NCQ scaling [66-72]. Although more hadrons are produced from fragmentation than from coalescence in this intermediate $p_{T}$ region (see Fig. 2), $v_{2}$ of $p(\pi, K)$ from coalescence is about a factor of $4(2,3)$ larger than that from fragmentation (see Fig. 5). Coalescence, therefore, still contributes significantly to the final $v_{2}$ of all hadrons. Without coalescence (dashed lines in Fig. 4), COLBT-hydro underestimates the $v_{2}$ by up to a factor of 2 in this $p_{T}$ region. The hadronic cascade further pushes hadrons, especially baryons, toward higher $p_{T}$, increasing both $R_{A A}$ and $v_{2}$ of identified particles in agreement with the experimental data at intermediate $p_{T}$. COLBT-hydro also predicts a slightly larger $v_{2}$ of $K$ than $\pi$

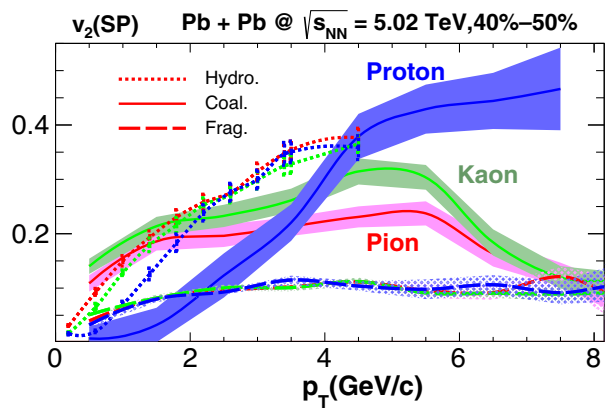

FIG. 5. COLBT-hydro results on $v_{2}\left(p_{T}\right)$ for $\pi, K$, and $p$ from hydro freeze-out (dotted), parton coalescence (solid), and fragmentation (dashed) in $40 \%-50 \% \mathrm{~Pb}+\mathrm{Pb}$ collisions at $\sqrt{s_{N N}}=5.02 \mathrm{TeV}$. 


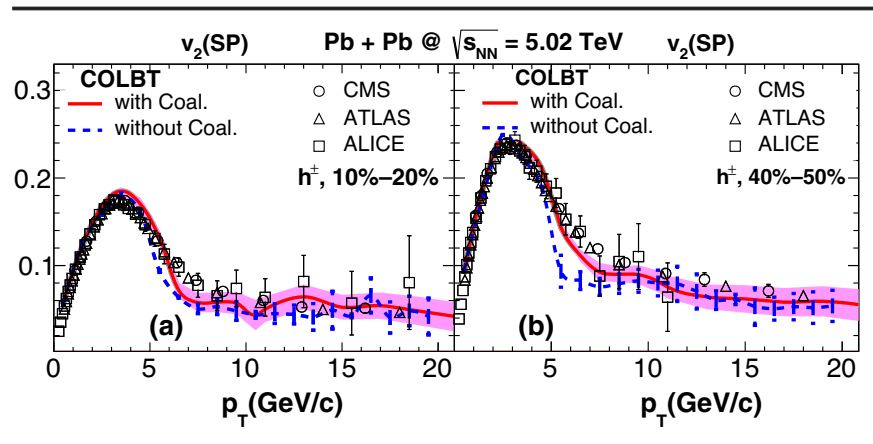

FIG. 6. COLBT-hydro results on $v_{2}\left(p_{T}\right)$ of charged hadrons in (a) $10 \%-20 \%$ and (b) $40 \%-50 \% \mathrm{~Pb}+\mathrm{Pb}$ collisions at $\sqrt{s_{N N}}=$ $5.02 \mathrm{TeV}$ with (solid) and without quark coalescence (dashed) compared with experimental data [27-29].

in this region because of the enhanced thermal strangeness in parton coalescence.

Finally, we show $v_{2}\left(p_{T}\right)$ of all charged hadrons in $10 \%-20 \%$ and $40 \%-50 \% \mathrm{~Pb}+\mathrm{Pb}$ collisions as compared to experimental data [27-29] in Fig. 6. At high $p_{T}>$ $10 \mathrm{GeV} / c$, fragmentation dominates the hadron production, and $v_{2}$ for all light flavor hadrons becomes the same and arises from the geometric anisotropy of the medium and length dependence of the parton energy loss. Since the radiative parton energy loss $\Delta E$ has a logarithmic energy dependence and given the power-law behavior $1 / p_{T}^{n}$ of the initial jet spectra, $R_{A A} \approx 1-n \Delta E / p_{T}$ eventually becomes 1 and $v_{2}$ approaches to 0 at high $p_{T}$. LBT without thermal-shower coalescence can describe this trend well [54,91]. COLBT-hydro with the hydro-coal-frag hadronization and hadron cascade can, therefore, consistently describe both $R_{A A}\left(p_{T}\right)$ and $v_{2}\left(p_{T}\right)$ of charged hadrons in the whole $p_{T}$ range. As shown in Figs. 1 and 6, COLBThydro without quark coalescence significantly underestimates both $R_{A A}$ and $v_{2}\left(p_{T}\right)$ at the intermediate $p_{T}$ region. We have carried out similar studies in other collisions systems at both RHIC and LHC energies. The combined approach in the COLBT-hydro model can also describe the colliding energy dependence well. These studies with careful analyses of model uncertainties will be presented in a future publication.

Summary.-We carried out the first study of hadron spectra in $A+A$ collisions that combines the state-ofthe-art COLBT-hydro, the hydro-coal-frag hybrid hadronization, and hadron cascade. We demonstrated that the interplay between hydro freeze-out at low $p_{T}$, parton coalescence at intermediate $p_{T}$, and fragmentation at high $p_{T}$ can simultaneously explain the nuclear modification $R_{A A}$, elliptic anisotropy $v_{2}$ of charged and identified hadrons and their flavor dependence in the full range of $p_{T}$. The long-standing $R_{A A} \otimes v_{2}$ puzzle is solved for the first time with the inclusion of quark coalescence in this coupled approach, which significantly increases $v_{2}$ in the intermediate $p_{T}$ region. The predicted splitting between $v_{2}$ of $K$ and $\pi$ in the intermediate $p_{T}$ region can serve as a precision test for the coalescence mechanism. Parton coalescence has been shown to be essential for explaining the NCQ scaling of $v_{2}$ at intermediate $p_{T}$ irrespective of the details of the coalescence models [66-78]. This should also be the case for our combined approach with hydro-coalfrag hybrid hadronization and hadron cascade to solve the $R_{A A} \otimes v_{2}$ puzzle.

We thank S. Cao, Y. He, C.-M. Ko, L. Pang, G. Qin, H. Song, and Z. Yang for discussions. This work was supported in part by NSFC under Grants No. 11935007, No. 11221504, No. 11861131009, and No. 11890714, the Fundamental Research Funds for Central Universities in China, U.S. DOE under Grant No. DE-AC02-05CH11231, U.S. NSF under Grant No. ACI-1550300 and No. OAC2004571, EU ERDF and H2020 Grant No. 82409, ERC Grant No. ERC-2018-ADG-835105, Spanish AEI Grant No. FPA2017-83814-P and MDM-2016-0692, Xunta de Galicia Research Center accreditation 2019-2022, and the UCB-CCNU Collaboration Grant. Computations are performed at NSC3/CCNU.

*xnwang@lbl.gov

†Present address: Nuclear Science Division MS 70R0319, Lawrence Berkeley National Laboratory, Berkeley, California 94720, USA.

[1] M. Gyulassy and L. McLerran, Nucl. Phys. A750, 30 (2005).

[2] J. Adams et al. (STAR Collaboration), Nucl. Phys. A757, 102 (2005).

[3] K. Adcox et al. (PHENIX Collaboration), Nucl. Phys. A757, 184 (2005).

[4] P. Jacobs and X.-N. Wang, Prog. Part. Nucl. Phys. 54, 443 (2005).

[5] B. Muller, J. Schukraft, and B. Wyslouch, Annu. Rev. Nucl. Part. Sci. 62, 361 (2012).

[6] K. H. Ackermann et al. (STAR Collaboration), Phys. Rev. Lett. 86, 402 (2001).

[7] K. Adcox et al. (PHENIX Collaboration), Phys. Rev. Lett. 89, 212301 (2002).

[8] K. Aamodt et al. (ALICE Collaboration), Phys. Rev. Lett. 105, 252302 (2010).

[9] G. Aad et al. (ATLAS Collaboration), Phys. Lett. B 707, 330 (2012).

[10] S. Chatrchyan et al. (CMS Collaboration), Eur. Phys. J. C 72, 2012 (2012).

[11] K. Adcox et al. (PHENIX Collaboration), Phys. Rev. Lett. 88, 022301 (2001).

[12] C. Adler et al. (STAR Collaboration), Phys. Rev. Lett. 89, 202301 (2002).

[13] X.-N. Wang, Nucl. Phys. A750, 98 (2005).

[14] K. Aamodt et al. (ALICE Collaboration), Phys. Lett. B 696, 30 (2011).

[15] A. Majumder and M. Van Leeuwen, Prog. Part. Nucl. Phys. 66, 41 (2011).

[16] P. Romatschke and U. Romatschke, Phys. Rev. Lett. 99, 172301 (2007). 
[17] H. Song, S. A. Bass, U. Heinz, T. Hirano, and C. Shen, Phys. Rev. Lett. 106, 192301 (2011); 109, 139904(E) (2012).

[18] C. Gale, S. Jeon, B. Schenke, P. Tribedy, and R. Venugopalan, Phys. Rev. Lett. 110, 012302 (2013).

[19] P. Huovinen, Int. J. Mod. Phys. E 22, 1330029 (2013).

[20] U. Heinz and R. Snellings, Annu. Rev. Nucl. Part. Sci. 63, 123 (2013).

[21] C. Gale, S. Jeon, and B. Schenke, Int. J. Mod. Phys. A 28, 1340011 (2013).

[22] H. Niemi, K. J. Eskola, and R. Paatelainen, Phys. Rev. C 93, 024907 (2016).

[23] J. E. Bernhard, J. S. Moreland, S. A. Bass, J. Liu, and U. Heinz, Phys. Rev. C 94, 024907 (2016).

[24] S. McDonald, C. Shen, F. Fillion-Gourdeau, S. Jeon, and C. Gale, Phys. Rev. C 95, 064913 (2017).

[25] W. Zhao, H.-j. Xu, and H. Song, Eur. Phys. J. C 77, 645 (2017).

[26] J. Adams et al. (STAR Collaboration), Phys. Rev. Lett. 92, 052302 (2004).

[27] A. M. Sirunyan et al. (CMS Collaboration), Phys. Lett. B 776, 195 (2018).

[28] M. Aaboud et al. (ATLAS Collaboration), Eur. Phys. J. C 78, 997 (2018).

[29] S. Acharya et al. (ALICE Collaboration), J. High Energy Phys. 07 (2018) 103.

[30] W.-J. Xing, S. Cao, G.-Y. Qin, and H. Xing, Phys. Lett. B 805, 135424 (2020).

[31] Y.-F. Liu, W.-J. Xing, X.-Y. Wu, G.-Y. Qin, S. Cao, and H. Xing, arXiv:2107.01522.

[32] X.-N. Wang and M. Gyulassy, Phys. Rev. Lett. 68, 1480 (1992).

[33] X.-N. Wang, Phys. Rev. C 61, 064910 (2000).

[34] I. Vitev and M. Gyulassy, Phys. Rev. Lett. 89, 252301 (2002).

[35] E. Wang and X.-N. Wang, Phys. Rev. Lett. 89, 162301 (2002).

[36] K. J. Eskola, H. Honkanen, C. A. Salgado, and U. A. Wiedemann, Nucl. Phys. A747, 511 (2005).

[37] G.-Y. Qin, J. Ruppert, C. Gale, S. Jeon, G. D. Moore, and M. G. Mustafa, Phys. Rev. Lett. 100, 072301 (2008).

[38] B. Schenke, C. Gale, and S. Jeon, Phys. Rev. C 80, 054913 (2009).

[39] X.-F. Chen, T. Hirano, E. Wang, X.-N. Wang, and H. Zhang, Phys. Rev. C 84, 034902 (2011).

[40] A. Majumder and C. Shen, Phys. Rev. Lett. 109, 202301 (2012).

[41] A. Buzzatti and M. Gyulassy, Phys. Rev. Lett. 108, 022301 (2012).

[42] K. C. Zapp, F. Krauss, and U. A. Wiedemann, J. High Energy Phys. 03 (2013) 080.

[43] M. Gyulassy, I. Vitev, and X. N. Wang, Phys. Rev. Lett. 86, 2537 (2001).

[44] A. Kumar, E. Bianchi, J. Elledge, A. Majumder, G.-Y. Qin, and C. Shen, Nucl. Phys. A967, 536 (2017).

[45] D. Zigic, B. Ilic, M. Djordjevic, and M. Djordjevic, Phys. Rev. C 101, 064909 (2020).

[46] Y. Mehtar-Tani, J. G. Milhano, and K. Tywoniuk, Int. J. Mod. Phys. A 28, 1340013 (2013).
[47] G.-Y. Qin and X.-N. Wang, Int. J. Mod. Phys. E 24, 1530014 (2015).

[48] J.-P. Blaizot and Y. Mehtar-Tani, Int. J. Mod. Phys. E 24, 1530012 (2015).

[49] D. Molnar and D. Sun, arXiv:1305.1046.

[50] J. Noronha-Hostler, B. Betz, J. Noronha, and M. Gyulassy, Phys. Rev. Lett. 116, 252301 (2016).

[51] X. Zhang and J. Liao, arXiv:1311.5463.

[52] J. Liao and E. Shuryak, Phys. Rev. Lett. 102, 202302 (2009).

[53] B. Z. Kopeliovich, J. Nemchik, I. K. Potashnikova, and I. Schmidt, Phys. Rev. C 86, 054904 (2012).

[54] S. Cao, L.-G. Pang, T. Luo, Y. He, G.-Y. Qin, and X.-N. Wang, Nucl. Part. Phys. Proc. 289-290, 217 (2017).

[55] C. Andres, N. Armesto, H. Niemi, R. Paatelainen, and C. A. Salgado, Phys. Lett. B 803, 135318 (2020).

[56] J. Xu, J. Liao, and M. Gyulassy, Chin. Phys. Lett. 32, 092501 (2015).

[57] S. Shi, J. Liao, and M. Gyulassy, Chin. Phys. C 42, 104104 (2018).

[58] S. Shi, J. Liao, and M. Gyulassy, Chin. Phys. C 43, 044101 (2019).

[59] K. Werner, I. Karpenko, M. Bleicher, T. Pierog, and S. Porteboeuf-Houssais, Phys. Rev. C 85, 064907 (2012).

[60] I. P. Lokhtin, A. V. Belyaev, L. V. Malinina, S. V. Petrushanko, E. P. Rogochaya, and A. M. Snigirev, Eur. Phys. J. C 72, 2045 (2012).

[61] G. Aad et al. (ATLAS Collaboration), Phys. Rev. Lett. 111, 152301 (2013).

[62] K. C. Zapp, Phys. Lett. B 735, 157 (2014).

[63] J. Adam et al. (ALICE Collaboration), Phys. Lett. B 753, 511 (2016).

[64] Y. He, S. Cao, W. Chen, T. Luo, L.-G. Pang, and X.-N. Wang, Proc. Sci., HardProbes2018 (2019) 100.

[65] B. Betz, M. Gyulassy, and G. Torrieri, Phys. Rev. C 84, 024913 (2011).

[66] R. J. Fries, B. Muller, C. Nonaka, and S. A. Bass, Phys. Rev. Lett. 90, 202303 (2003).

[67] R. J. Fries, B. Muller, C. Nonaka, and S. A. Bass, Phys. Rev. C 68, 044902 (2003).

[68] V. Greco, C. M. Ko, and P. Levai, Phys. Rev. Lett. 90, 202302 (2003).

[69] V. Greco, C. M. Ko, and P. Levai, Phys. Rev. C 68, 034904 (2003).

[70] V. Greco, C. M. Ko, and R. Rapp, Phys. Lett. B 595, 202 (2004).

[71] D. Molnar and S. A. Voloshin, Phys. Rev. Lett. 91, 092301 (2003).

[72] R. C. Hwa and C. B. Yang, Phys. Rev. C 70, 024905 (2004).

[73] J. Adams et al. (STAR Collaboration), Phys. Rev. C 72, 014904 (2005).

[74] J. Adams et al. (STAR Collaboration), Phys. Rev. Lett. 95, 122301 (2005).

[75] A. Adare et al. (PHENIX Collaboration), Phys. Rev. Lett. 98, 162301 (2007).

[76] B. I. Abelev et al. (STAR Collaboration), Phys. Lett. B 655, 104 (2007).

[77] A. Adare et al. (PHENIX Collaboration), Phys. Rev. C 85, 064914 (2012). 
[78] A. Adare et al. (PHENIX Collaboration), Phys. Rev. C 83, 064903 (2011).

[79] C. Shen, U. Heinz, P. Huovinen, and H. Song, Phys. Rev. C 82, 054904 (2010).

[80] S. Ryu, J.-F. Paquet, C. Shen, G. Denicol, B. Schenke, S. Jeon, and C. Gale, Phys. Rev. C 97, 034910 (2018).

[81] A. G. Knospe, C. Markert, K. Werner, J. Steinheimer, and M. Bleicher, Phys. Rev. C 104, 054907 (2021).

[82] W. Chen, S. Cao, T. Luo, L.-G. Pang, and X.-N. Wang, Phys. Lett. B 810, 135783 (2020).

[83] W. Chen, S. Cao, T. Luo, L.-G. Pang, and X.-N. Wang, Phys. Lett. B 777, 86 (2018).

[84] Z. Yang, W. Chen, Y. He, W. Ke, L. Pang, and X.-N. Wang, Phys. Rev. Lett. 127, 082301 (2021).

[85] L. Pang, Q. Wang, and X.-N. Wang, Phys. Rev. C 86, 024911 (2012).

[86] L.-G. Pang, H. Petersen, and X.-N. Wang, Phys. Rev. C 97, 064918 (2018).

[87] X.-N. Wang and Y. Zhu, Phys. Rev. Lett. 111, 062301 (2013).

[88] H. Li, F. Liu, G.-1. Ma, X.-N. Wang, and Y. Zhu, Phys. Rev. Lett. 106, 012301 (2011).

[89] Y. He, T. Luo, X.-N. Wang, and Y. Zhu, Phys. Rev. C 91, 054908 (2015); 97, 019902(E) (2018).

[90] S. Cao, T. Luo, G.-Y. Qin, and X.-N. Wang, Phys. Rev. C 94, 014909 (2016).

[91] S. Cao, T. Luo, G.-Y. Qin, and X.-N. Wang, Phys. Lett. B 777, 255 (2018).

[92] X.-F. Guo and X.-N. Wang, Phys. Rev. Lett. 85, 3591 (2000).

[93] X.-N. Wang and X.-F. Guo, Nucl. Phys. A696, 788 (2001).

[94] B.-W. Zhang and X.-N. Wang, Nucl. Phys. A720, 429 (2003).

[95] A. Schafer, X.-N. Wang, and B.-W. Zhang, Nucl. Phys. A793, 128 (2007).

[96] T. Luo, S. Cao, Y. He, and X.-N. Wang, Phys. Lett. B 782, 707 (2018).

[97] W. Chen, S. Cao, T. Luo, L.-G. Pang, and X.-N. Wang, Nucl. Phys. A1005, 121934 (2021).

[98] A. Luo, Y.-X. Mao, G.-Y. Qin, E.-K. Wang, and H.-Z. Zhang, arXiv:2109.14314.
[99] A. Bazavov et al. (HotQCD Collaboration), Phys. Rev. D 90, 094503 (2014).

[100] J. S. Moreland, J. E. Bernhard, and S. A. Bass, Phys. Rev. C 92, 011901(R) (2015).

[101] The plateau width is $\eta_{\text {flat }}$ at $=2.0 \mathrm{fm}$ and the Gaussian falloff width is $\sigma_{\eta}=1.8 \mathrm{fm}$.

[102] T. Sjostrand, S. Mrenna, and P. Z. Skands, Comput. Phys. Commun. 178, 852 (2008).

[103] K. J. Eskola, P. Paakkinen, H. Paukkunen, and C. A. Salgado, Eur. Phys. J. C C77, 163 (2017).

[104] W. Zhao, C. M. Ko, Y.-X. Liu, G.-Y. Qin, and H. Song, Phys. Rev. Lett. 125, 072301 (2020).

[105] In principle, the energy and momentum of the coalesced thermal partons with $p_{T}<1.5 \mathrm{GeV} / c$ should be subtracted from the bulk medium to conserve the total energy and momentum. However, we find the effect is negligible in the final hadron spectra, which we will neglect.

[106] We tune the Lund string parameter probstoUD $=0.4$, which controls the suppression of $s$ quark pair production relative to $u, d$ quarks in the string fragmentation, to reproduce the $K / \pi$ ratio in minimum-bias $p-p$ collisions at $\sqrt{s}=5.02 \mathrm{TeV}$ [107].

[107] S. Acharya et al. (ALICE Collaboration), Phys. Rev. C 99, 024906 (2019).

[108] A. Kumar et al. (JETSCAPE Collaboration), Phys. Rev. C 102, 054906 (2020).

[109] S. A. Bass et al., Prog. Part. Nucl. Phys. 41, 255 (1998).

[110] M. Bleicher, E. Zabrodin, C. Spieles, S. A. Bass, C. Ernst, S. Soff, L. Bravina, M. Belkacem, H. Weber, H. Stöcker, and W. Greiner, J. Phys. G 25, 1859 (1999).

[111] Experimental data on the hadron spectra in $p+p$ collision at $\sqrt{s_{N N}}=5.02 \mathrm{TeV}$ [112] are used as the baseline for the model calculations of $R_{A A}$.

[112] S. Acharya et al. (ALICE Collaboration), J. High Energy Phys. 11 (2018) 013.

[113] S. Acharya et al. (ALICE Collaboration), Phys. Rev. C 101, 044907 (2020).

[114] S. Acharya et al. (ALICE Collaboration), J. High Energy Phys. 09 (2018) 006. 